\section{Als Tumor imponierende primäre pulmonale Kryptokokkose bei einer immunkompetenten Patientin. Ein Beitrag zur Differentialdiagnose}

M. J. Amthor ${ }^{1}$, S. Bontikous ${ }^{1}$, G. Pasterkamp ${ }^{2}$, T. Schaberg ${ }^{3}$

${ }^{1}$ Pathologisches Institut (Chefarzt: Prof. M. J. Amthor), Diakoniekrankenhaus Rotenburg (Wümme)

${ }^{2}$ Gemeinschaftspraxis $f$. Lungenheilkunde, Stade

${ }^{3}$ Lungenklinik (Chefarzt: Prof. T. Schaberg), Diakoniekrankenhaus Rotenburg (Wümme)
Zusammenfassung: Die vorliegende Kasuistik beschreibt eine primäre pulmonale Kryptokokkose der Bronchusschleimhaut bei einer 78 Jahre alten immunkompetenten Patientin. Der Röntgenbefund imponierte zunächst als Tumor. Der Erreger konnte erst nach wiederholten bioptischen Versuchen in der nekrotischen Schleimhaut nachgewiesen werden. Hinweise auf eine Dissemination bestanden nicht. Die Therapie erfolgte mit Fluconazol.

Primary Pulmonary Cryptococcosis Suspicious of Lung Cancer in an Immunocompetent Female Patient. A Contricution to Differential Diagnosis: Case report of a primary cryptococcosis of the lung in a 78-year old non-immunocompromised female. The patient presented with a mass in the right upper lobe, highly suspicious of lung cancer. Cryptococcus finally was detected on repeated biopsies from ulcerated and necrotic bronchial mucosa. A clinical work-up showed no evidence of dissemination and no signs of immunoinsufficiency. Mass reduction in the lung was achieved under therapy with fluconazol.

\section{Einleitung}

Während die Kryptokokkose bei Patienten bei Immunschwäche infolge einer Immunsuppression oder AIDS die zweithäufigste systemische Pilzinfektion darstellt, ist die Kryptokokkose als primäre Erkrankung ausgesprochen selten [1]. Der Erreger (Cryptococcus neoformans) ist ein weltweit verbreiteter Pilz. Die Infektion erfolgt durch Inhalation sporenhaltiger Stäube, wobei insbesondere Taubenkot Sporen enthält. Der Erreger, Cryptococcus neoformans, gilt als fakultativ pathogen. Er hat einen Durchmesser von 4 bis 20 Mikrometer und bildet eine charakteristische Kapsel aus Polysacchariden. Etwa $1 / 3$ der Erkrankten bleibt asymptomatisch [1]. Die übrigen Patienten entwickeln ein unterschiedlich ausgeprägtes Krankheitsbild mit Husten und Fieber. Von der durch den Kryptokokkus induzierten knotigen, tumorartigen Gewebsreaktion, leitet sich auch das Attribut „neoformans“ ab. Gefürchtet ist die Kryptokokkenmeningitis mit einer Mortalität von $7 \%$, bei unbehandelten Patienten von nahezu $100 \%[1,2]$. Wir berichten über eine 78 Jahre alte, bis

Pneumologie 2000; 54: 58-60

(c) Georg Thieme Verlag Stuttgart · New York ISSN 0934-8387 dahin gesunde Patientin, bei der lange der Verdacht auf ein Bronchialkarzinom bestand.

\section{Fallbericht}

Die 78 Jahre alte Patientin klagte seit etwa 7 Monaten über vermehrt Husten ohne Auswurf oder Hämoptysen sowie über Luftnot bei stärkerer Belastung. Aus der Anamnese sind ein arterieller Hypertonus und eine kompensierte Niereninsuffizienz bekannt. Im Röntgenbild konnte ein tumorartiges Infiltrat im rechten Lungenoberlappen nachgewiesen werden (Abb.1). Die daraufhin erfolgte stationäre Untersuchung in einer Medizinischen Klinik erbrachte jedoch keinen Tumornachweis. Histologisch bestand lediglich eine unspezifische, ulzeröse Bronchitis. Da nach Entlassung keine klinische Besserung und keine Rückbildung des Infiltrates erfolgten, wurde 6 Monate nach Entlassung noch einmal eine ambulante Bronchoskopie mit Entnahme von Gewebsproben durchgeführt. In diesen Gewebsproben konnten schließlich Kryptokokken nachgewiesen werden. Die Patientin wurde daraufhin wieder in einer Lungenklinik stationär aufgenommen. In Röntgenbildern des Thorax sowie im Computertomogramm zeigte sich im rechten Oberlappen ein im Durchmesser $4 \mathrm{~cm}$ großes inhomogenes Infiltrat mit fraglicher Verkalkung ohne Nachweis einer Lymphadenopathie (Abb. 2). Lungenfunktionell keine Ventilationsstörung, keine Diffusionsstörung. Die üblichen Laborparameter lagen im Bereich der Norm, HIV-1 und HIV-2 waren im ELISA-Test negativ. CD-

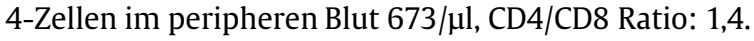

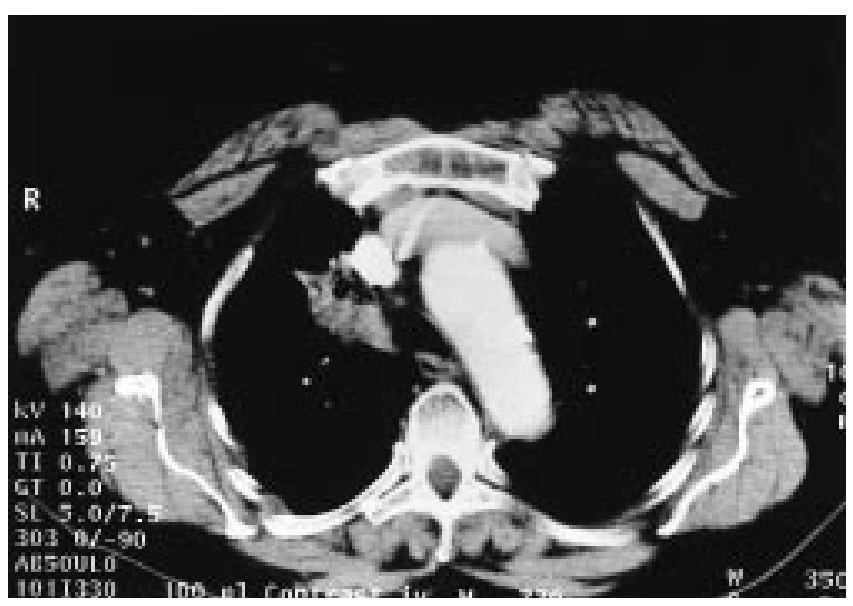

Abb. 1 Thoraxübersicht vor Therapie mit einem tumorverdächtigen Infiltrat im rechten Oberlappen. 


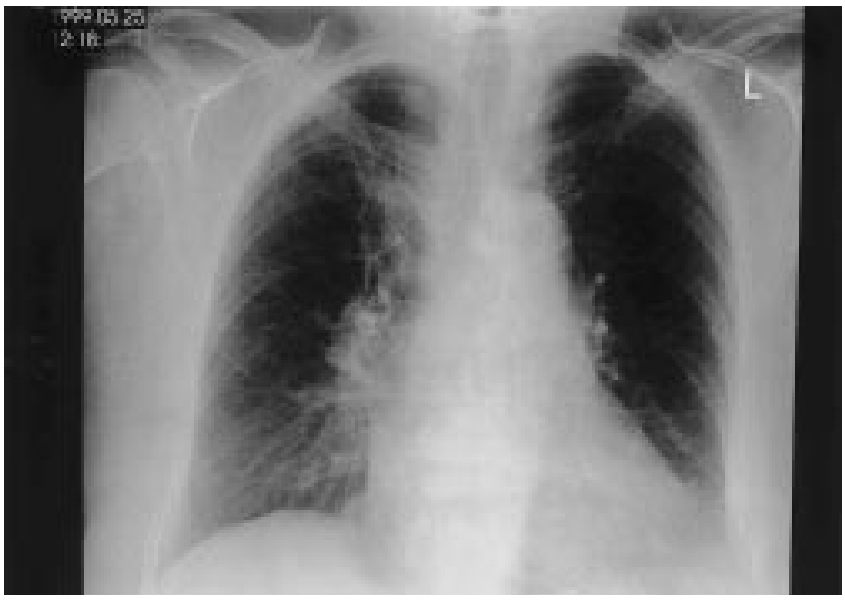

Abb. 2 Computertomogramm vor Therapie mit Darstellung des tumorverdächtigen Infiltrates.

\section{Histologischer Befund}

Zur Untersuchung kamen zahlreiche, zusammen $0,7 \mathrm{~cm}$ im Durchmesser große Gewebsproben. Mikroskopisch waren die Bronchuswandstrukturen durch Nekrosen partiell zerstört. Im Randbereich der Nekrosen ein demarkierendes, z.T. bereits vernarbtes Granulationsgewebe mit Infiltraten aus Lymphozyten, Plasmazellen und Granulozyten. Auf der ulzerierten Schleimhautoberfläche ein fibrinös-granulozytäres Exsudat. In der Schleimhaut bereits im HE-Präparat nachweisbare (Abb.3) runde und ovale Kryptokokken mit einem Durchmesser von 4 bis 20 Mikrometern und basophiler Kapsel, die sich im dann angefertigten Alcianblau-Präparat (Abb.4) als typische strahlenkranzartige Schleimkapsel darstellte. Die zur Sicherheit angefertigten Ziehl-Neelsen-Präparate waren ohne Nachweis säurefester Stäbchen. Tumorgewebe war nicht nachweisbar.

\section{Verlauf}

Die Therapie erfolgt mit Fluconazol, wobei im vorliegenden Fall die Dosis wegen einer vorbestehenden Niereninsuffizienz 2. Grades (Ansteigen der Kreatininwerte) auf $200 \mathrm{mg} / \mathrm{die}$ reduziert werden musste. Die Kontrolle nach insgesamt 2 Monaten Behandlung zeigt eine Rückbildung des Infiltrates im rechten apikalen Oberlappensegment. Wegen eines blutenden Magenulkus musste die Therapie mit Fluconazol nach insgesamt zwei Monaten jedoch auf Wunsch der Patientin abgesetzt werden. Da die Patientin auch gegenwärtig einer weiteren Therapie ablehnend gegenüber steht, wurde vereinbart, unter regelmäßigen Röntgenkontrollen den Spontanverlauf abzuwarten.

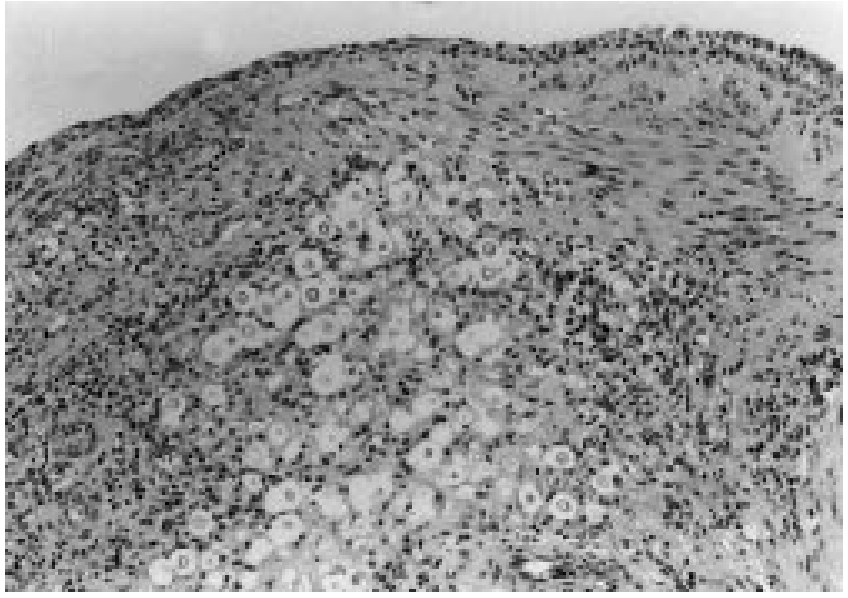

Abb. 3 Bronchusschleimhaut mit massenhaft eingeschlossenen Kryptokokken. Gut erkennbar sind die zystischen Erreger mit dem hellen Hof. HE-Färbung 100-fach.

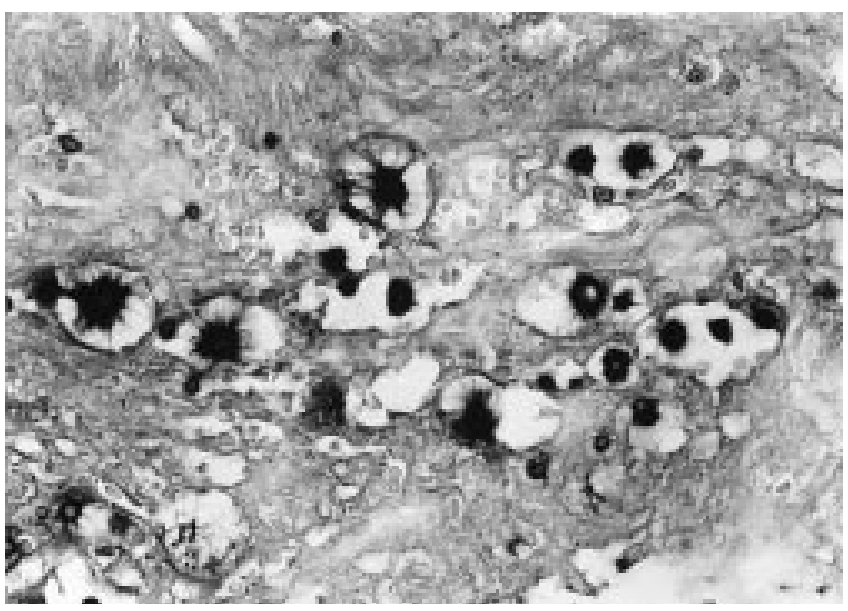

Abb. 4 Detailaufnahme nach Alcianblau-Färbung zur Darstellung des typischen Strahlenkranzes. Alcianblau-Färbung, 400-fach (Ölimmersion).

\section{Diskussion}

Die pulmonale Kryptokokkose stellt bei immunkompromitierten Patienten die zweithäufigste Pilzinfektion dar [1,3]. Die radiologischen Erscheinungsformen der pulmonalen Kryptokokkose sind unterschiedlich, sie reichen von tumorartigen Formationen (deshalb der Speziesname „neoformans“) bis hin zu dem Bild einer Miliartuberkulose [4]. Die Kryptokokkose bei immunkompetenten Menschen ist dagegen sehr selten $[1,2,5,6)$. Da in diesen Fällen keine Vorerkrankungen bekannt sind, stellt sich hier bei einer pulmonalen Infektion zunächst einmal der Verdacht auf ein Bronchialkarzinom, welches bioptisch abgeklärt werden muss. Dabei steht die Kryptokokkose als Ursache derartiger Infiltrate zunächst sicherlich nicht im Vordergrund der differentialdiagnostischen Überlegungen. Entscheidend ist das Ergebnis der histologischen Diagnostik, welche die gesamte Differentialdiagnose einer ulzerösen nekrotisierenden Bronchitis durcharbeiten muss. Sie umfasst das große Spektrum, welches von vollständigen Tumornekrosen über Vaskulitiden (z.B. M. Wegener), allergische Reaktionen bis hin zu infektiösen Nekrosen reicht $[7,8]$. Dabei steht 
in Europa die Tuberkulose immer noch an erster Stelle als Ursache infektiös bedingter Nekrosen. Bei der Wertung der einzelnen mikroskopischen Phänomene muss immer bedacht werden, dass wesentliche und richtungweisende Befunde bei der Biopsie nicht erfasst oder nur in wenigen Partikeln nachweisbar sein können. So hat auch im vorliegenden Fall erst die wiederholte Gewebsentnahme den Erreger nachweisen können, wobei dieser dann aber bereits im routinemäßig erstellten HE-Präparat zweifelsfrei zu identifizieren war. Die Darstellung der Kapsel durch Alcianblau zeigt dann den typischen intensiv blau angefärbten Stahlenkranz. Die sonst zum Pilznachweis benutzte Darstellung mit MethaminSilber (Grocott-Färbung) ist zum Nachweis des Cryptococcus neoformans weniger geeignet, da die Schleimsubstanzen den eigentlichen Erreger einhüllen und somit die Imprägnierung durch das Silber behindern.

\section{Literatur}

${ }^{1}$ Cox GM, Perfect JR. Microbiology and epidemiology of cryptococcal infection. In: UpToDate Inc. Wellesly MA Mass. USA (CDROM): 1999; 7: 1 - 3

2 Schaberg T, Mai J, Seibold M, Staib F. Lungenkryptokokkom - ein Beitrag zur Diagnostik und Therapie. Internist 1988; 29: $510-$ 515

${ }^{3}$ Wasser L, Talavera W. Pulmonary cryptococcosis in AIDS. Chest 1987; 92: $692-695$

${ }^{4}$ Amthor M, Arents B, Bartelheimer E. Kryptokokkensepsis unter dem Bild einer Miliartuberkulose bei malignem Lymphom. Pneumologie 1991; 45: 110 - 113

${ }^{5}$ Caballes RL, Caballes Jr RA. Primary cryptococcal prostatitis in an apparently uncompromised host prostata. 1999; 39: 119 - 122

${ }^{6}$ Kerkering TM, Turi MA. Pulmonary Cryptococcosis. In: Sarosi GA, Davis SF. Fungal diseases of the lung. 2nd Ed. New York: Raven Press, 1993

${ }^{7}$ Katzenstein A-LA. Infection II. Granulomatous infections. In: Katzensstein and Askin's surgical pathology of non-neoplastic lung disease. 3rd ed. Philadelphia: W. B. Saunders, 1997. 295 300

${ }^{8}$ Sobonya RE. Fungal diseases, including allergic bronchopulmonary aspergillosis. In: Thurlbeck WM, Churg AM (ed). Pathology of the lung 2nd ed. Stuttgart: Thieme, 1995. 317 - 324
Prof. Dr. Michael J. Amthor

Pathologisches Institut Diakoniekrankenhaus Postfach

27342 Rotenburg (Wümme) 